\title{
The LAGUNA project - towards the giant liquid based detectors for proton decay searches and for low energy neutrino astrophysics
}

\section{Jan Kisiel ${ }^{1}$}

Institute of Physics, University of Silesia

Uniwersytecka 4, 40-007 Katowice, Poland

E-mail: Jan.Kisiel@us.edu.pl

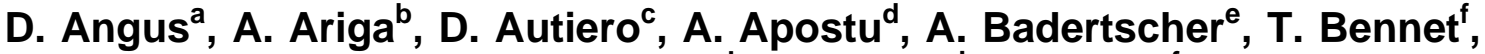
G. Bertola ${ }^{g}$, P.F. Bertola ${ }^{g}$, O. Besida ${ }^{h}$, A. Bettini', C. Booth ${ }^{f}$, J.L. Borne $^{c}$, I. Brancus $^{d}$, W. Bujakowski ${ }^{j}$, J.E. Campagne ${ }^{c}$, G. Cata-Danil ${ }^{d}$, F. Chipesiu ${ }^{d}$, M. Chorowski ${ }^{k}$, J. Cripps ${ }^{f}$, A. Curioni ${ }^{i}$, S. Davidson ${ }^{c}$, Y.R.A Declais ${ }^{c}, U$. Drost $^{\mathrm{g}}$, O. Duliu', J. Dumarchez ${ }^{\mathrm{c}}$, T. Enqvist ${ }^{\mathrm{m}}$, A. Ereditato ${ }^{\mathrm{b}}$, F. v Feilitzsch ${ }^{\mathrm{n}}$, H. Fynbo ${ }^{\circ}$, T. Gamble ${ }^{f}$, G. Galvanin ${ }^{p}$, A. Gendotti ${ }^{e}$, W. Gizicki ${ }^{k}$, M. GogerNeff $^{n}$, U. Grasslin ${ }^{g}$, D. Gurney ${ }^{q}$, M. Hakala ${ }^{r}$, S. Hannestad ${ }^{\circ}$, M. Haworth $^{q}$, A. Jipa', F. Juget ${ }^{b}$, T. Kalliokoskis, S. Katsanevas ${ }^{c}$, M. Keen', I.Kreslob, V. Kudryastev $^{f}$, P. Kuusiniemi ${ }^{m}$, L. Labarga ${ }^{\mathrm{u}}$, T. Lachenmaier ${ }^{\mathrm{n}}$, J.C. Lanfranchi $^{n}$, I. Lazanu', T. Lewke ${ }^{n}$, K. Loo ${ }^{m}$, P. Lightfoot ${ }^{f}$, M. Lindner ${ }^{v}$, P. Lombardi $^{\mathrm{w}}$, A. Longhin ${ }^{\mathrm{h}}$, J. Maalampi ${ }^{\mathrm{s}}$, M. Marafini ${ }^{\mathrm{c}}$, A. Marchionni ${ }^{\mathrm{e}}$, R.M. Margineanu $^{d}$, A. Markiewicz ${ }^{\mathrm{x}}$, T. Marrodan-Undagoita ${ }^{n}$, J.E. Marteau ${ }^{\mathrm{c}}$, R. Matikainen $^{\mathrm{r}}$, Q. Meindl ${ }^{\mathrm{n}}$, M. Messina ${ }^{\mathrm{b}}$, J.W. Mietelski ${ }^{\mathrm{y}}$, B. Mitrica ${ }^{\mathrm{d}}$, A. Mordasini $^{g}$, L. Mosca ${ }^{h}$, U. Moser $^{b}$, G. Nuijten ${ }^{r}$, L. Oberauer ${ }^{n}$, A. Oprina ${ }^{d}$, S. Paling $^{f}$, S. Pascoli ${ }^{\mathrm{a}}$, T. Patzak ${ }^{\mathrm{c}}$, M. Pectu ${ }^{\mathrm{d}}$, Z. Pilecki ${ }^{\mathrm{j}}$, F. Piquemal ${ }^{\mathrm{c}}, \mathbf{W}$. Potzel $^{n}$, W. Pytel ${ }^{x}$, M. Raczynski ${ }^{x}$, G. Raffelt ${ }^{z}$, G. Ranucci ${ }^{w}$, G. Ristaino ${ }^{p}$, M. $^{2}$

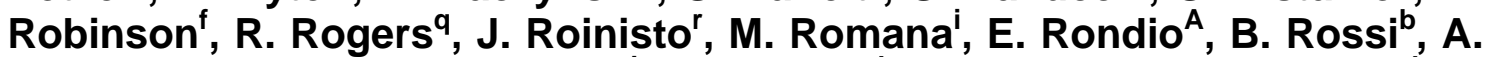
Rubbia $^{\mathrm{e}}$, Z. Sadecki ${ }^{\mathrm{x}}$, C. Saenz', A. Saftoiu ${ }^{\mathrm{d}}$, J. Salmelainen ${ }^{\mathrm{r}}$, O. Sima', J. Slizowski ${ }^{j}$, K. Slizowski ${ }^{j}$, J. Sobczyk ${ }^{B}$, N. Spooner ${ }^{f}$, S. Stoica ${ }^{d}$, J. Suhonens, R. Sulej ${ }^{A}$, M. Szarska ${ }^{y}$, T. Szeglowski ${ }^{C}$, M. Temussi ${ }^{p}$, J. Thompson ${ }^{q}$, L.

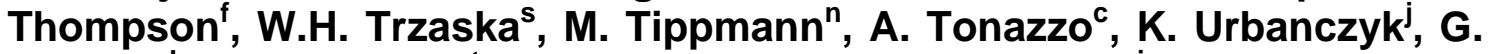
Vasseur $^{\mathrm{h}}$, A. Williams ${ }^{\mathrm{t}}$, J. Winter ${ }^{\mathrm{n}}, \mathrm{K}$. Wojtuszewska ${ }^{\mathrm{j}}$, M. Wurm ${ }^{\mathrm{n}}, \mathrm{A}$. Zalewska $^{\mathrm{y}}$, M. Zampaoloc ${ }^{\mathrm{c}}$, M. Zito ${ }^{\mathrm{h}}$

a) University of Durham (UDUR), University Office, Old Elvet, Durham DH1 3HP, United Kingdom

b) University of Bern, 4 Hochschulstrasse, $\mathrm{CH}-3012$, Bern

c) Centre National de la Recherche Scientifique, Institut National de Physique Nucléaire et de Physique des Particules (CNRS/IN2P3), 3 rue Michel-Ange, Paris 75794, France

d) Horia Hulubei National Institute of RD for Physics and Nuclear Engineering, IFIN-HH, 407 Atomistilor Street, R077125, Magurele, jud. ILFOV, PO Box MG-6, postal code RO-077125, Romania

e) ETH Zurich, 101 Raemistrasse, CH-8092 Zurich

Speaker 
f) The University of Sheffield (USFD), New Spring House 231, Glossop Road, Sheffield S102GW, United Kingdom g) Lombardi Engineering Limited, via R.Simen, CH-6648, Minusio

h) Commissariat à l' Energie Atomique (CEA)/ Direction des Sciences de la Matière, 25 rue Leblanc, Paris 75015 , France

i) Laboratorio Subterráneo de Canfranc (LSC), Plaza del Ayuntamiento no. 1, 22880 Canfranc (Huesca), Spain

j) Mineral and Energy Economy Research Institute of the Polish Academy of Sciences (IGSMIE-PAN), Wybickiego 7, 30-950 Kraków, Poland

k) Wroclaw University of Technology (PWr Wroclaw), ul. Wybrzeze Wyspianskiego 27, 50-370 Wroclaw, Poland l) University of Bucarest (UoB), Faculty of Physics Bld.Atomistilor nr.405, Physics Platform, Magurele, Ilfov County, RO-077125, MG-11 Bucharest-Magurele, Romania

m) University of Oulu (U-OULU), 1 Pentti Kaiteran Katu, Oulu 90014, Finland

n) Technische Universität München (TUM), 21 Arcisstrasse, München 80333, Germany

o) University of Aarhus (AU), 1 Norde Ringgade, Aarhus C 8000, Denmark

p) AGT Ingegneria Srl, Perugia, 10 A via della Pallotta, Perugia 06126, Italy

q) Technodyne International Ltd., Unit16, Shakespeare Business Centre Hathaway Close, Eastleigh UK SO 50 4SR, United Kingdom

r) Kalliosuunnittelu Oy Rockplan Ltd., 2 Asemamiehenkatu, Helsinki 00520, Finland

s) University of Jyväskylä (JyU), 9 Survontie, Jyväskylä 40014, Finland

t) Cleveland Potash Limited (CPL), Boulby Mine, Loftus, Saltburn Cleveland, TS13 4UZ, UK

u) Universidad Autónoma de Madrid (UAM), C/Einstein no. 1; Rectorado, Ciudad Universitaria de Cantoblanco, 28049 Madrid, Spain

v) Max-Planck-Institute for Nuclear Physics, Heidelberg

w) Istituto Nazionale di Fisica Nucleare, Sezione di Milano, Via Celoria 16, 20133, Milano, Italy

x) KGHM CUPRUM Ltd Research and Development Centre, Pl. 1 Maja, 50-136 Wroctaw, Poland

y) IFJ Pan, H.Niewodniczański Institute of Nuclear Physics PAN, Radzikowskiego 152, 31-342 Kraków, Poland

z) Max-Planck-Institute for Physics, Munich

A) High Energy Physics Department - A. Soltan Institute for Nuclear Studies (SINS) Hoza 69 00-681 Warsaw,

Poland

B) University of Wroclaw (UWr), Pl. Uniwersyetcki 1, 50-137 Wroclaw, Poland

C) University of Silesia, Institute of Physics, ul. Uniwersytecka 4, 40-007 Katowice, Poland

A next generation European deep underground neutrino observatory is considered within the LAGUNA design study. Three detector options are presently considered: GLACIER liquid argon Time Projection Chamber; LENA liquid scintillator and MEMPHYS water Cherenkov. It will provide both: the high statistics measurement of neutrinos from variety of sources, and high sensitivity searches for matter instability. To accommodate such giant detectors a new underground laboratory is required. The LAGUNA design study considers the following seven candidate sites in Europe: Boulby (UK), Canfranc (Spain), Fréjus (France/Italy), Pyhäsalmi (Finland), Polkowice-Sieroszowice (Poland), Slanic (Romania) and Umbria (Italy). The three detection techniques and summary of the physics potential of proposed detectors are discussed in this short paper.

European Physical Society Europhysics Conference on High Energy Physics EPS-HEP 2009,

Krakow , Poland

July 16-22, 200 


\section{Introduction}

Several important issues can be addressed to the next generation experiments with very large volume of active medium and signal to noise ratio guaranteed by their underground localization. The proton stability, the core-collapse supernova explosion mechanism, probing the Sun and the Earth interior and CP violation in the lepton sector certainly belong to such fundamental physics subjects. The candidate sites for a new European underground laboratory able to host a giant-size detector are identified in the LAGUNA design study. This brief paper presents three liquid-based detection techniques (liquid argon, liquid scintillator and water) and their physics potential. The details can be found in [1].

\section{Liquid-based detection techniques.}

GLACIER [2] is a 100 kton liquid argon (LAr) Time Projection Chamber (TPC) of the diameter of $70 \mathrm{~m}$ and height of $20 \mathrm{~m}$. The events can be reconstructed in 3D with excellent space resolution by exploiting the liquid ionization by charged particles and collection of scintillation and Cherenkov light. Very long (up to 20m) electron drift distance requires extremely high argon purity and the detector operation in the so-called bi-phase mode: electrons produced by ionization in the liquid phase will be extracted, by electric filed, to the gas phase and amplified. The single phase LAr TPC technique has been pioneered by the ICARUS collaboration [3].

LENA [4] is a 50 kton liquid scintillator tank of the length of $100 \mathrm{~m}$ and diameter of $26 \mathrm{~m}$, surrounded by $2 \mathrm{~m}$ of water for vetoing Cherenkov muons entering the inner part of the detector. The large detector radius requires an attenuation length of about $10 \mathrm{~m}$, which can be achieved by using e.g. PXE as scintillator solvent purified in an Aluminium-column, as has been shown by the BOREXINO collaboration [5]. The light produced by the scintillator will be collected by 12000 photomultipliers of $50 \mathrm{~cm}$ diameter each. This configuration corresponds to about $30 \%$ of the surface coverage.

MEMPHYS [6], an extrapolation of the Super-Kamiokande water Cerenkov detector [7], consists of 3-5 separate tanks 65m in diameter and 65m height each. Such dimensions meet the requirements of attenuation length of Cherenkov light and pressure of water on the bottom multipliers. Three tanks represent a total fiducial mass of $440 \mathrm{kton}$. Relativistic charged particles passing the water produce a cone of Cherenkov light which is imaged as a ring of photomultipliers covering the detector inner walls. A detector surface coverage of $30 \%$ can be obtained with about 81000 photomultipliers of $30 \mathrm{~cm}$ diameter per tank.

\section{Physics potential}

The broad physics program covered by these detectors includes: proton decay searches and studies of neutrinos produced in the: supernova explosion (also diffuse supernova neutrino background), Sun, atmosphere, Earth's interior (geo-neutrinos), nuclear reactors and particle accelerators beams. It is extensively discussed in [1]. Only proton decay and geo-neutrinos are briefly presented below.

In the Standard Model (SM) the proton is a stable particle, whereas in the Grand Unified Theories (GUTs) proton decays are predicted. Therefore, the observation of proton decay will be a further proof for the existence of physics beyond the SM. The majority of SUSY and non- 
SUSY models predict the proton partial lifetime $\tau_{p}$ at the level of $10^{33}-10^{37}$ years. This region will be accessible for detectors studied in LAGUNA. Here we discuss the expected limits at the 90\% C.L. only for two decay channels: (1) $p \rightarrow \pi^{0} e^{+}$and (2) $p \rightarrow v K^{+}$and 10 years of detector exposure (MEMPHYS mass equal to $500 \mathrm{kton}$ ). The $e^{+}$and two electromagnetic showers is a clear signature for (1) in the GLACIER LAr detector giving $\tau_{p} / B>0.4 \times 10^{35}$ years, where $B$ stands for the (unknown) branching ratio. A $938 \mathrm{MeV}$ energy signal from $\pi^{0}$ and $e^{+}$traveling in opposite directions will be evidence for (1). Three showering rings will identify (1) in water Cherenkov MEMPHYS detector allowing to reach $\tau_{p} / B>10^{35}$ years. The channel (2) is very clean in the GLACIER detector due to very precise $d E / d x$ and range identification of charged kaon and its decay products. This leads into a limit $\tau_{p} / B>0.6 \times 10^{35}$ years. The prompt monoenergetic kaon signal, together with various delayed signals from its decay products will identify (2) in the LENA detector. In case that no signal is observed during 10 years of detector exposure the $\tau_{p} / B$ is greater than $0.4 \times 10^{35}$ years. Since the $K^{+}$is below the Cherenkov threshold for channel (2) for MEMPHYS, the detection of kaon decay products is needed. The limit $\tau_{p} / B>$ $0.2 \times 10^{35}$ years can be obtained.

The beta-decays of uranium and thorium chains in the Earth interior originate electron antineutrinos, called geo-neutrinos. In the LENA detector, the inverse beta decay (IBD) reaction is the dominant channel for their detection. Assuming the detector location at Pyhäsalmi 1000 events/year is expected, with background of $\sim 240$ events/year of reactor neutrinos and $\sim 10$ events/year of radio purity background. Such high statistics measurement of geo-neutrinos flux can test geophysical models of the Earth. This number has to be compared to the first geoneutrinos observation by KamLAND collaboration, which reported a signal of $25 \pm 18$ events over 127 \pm 13 events of total background [8]. The observation of geo-neutrinos in MEMPHYS detector requires very challenging $2 \mathrm{MeV}$ threshold, whereas the threshold in LAr is too high for geo-neutrinos detection since no free protons are available.

\section{Acknowledgements}

The LAGUNA design study is financed by FP7 Research Infrastructure "Design Studies" Grant Agreement No. 212343 FP7-INFRA-2007-1.

\section{References}

[1] D. Autiero et. al., Large underground, liquid based detectors for astro-particle physics in Europe: scientific case and prospects, J. Cosmol. Astropart. Phys. 11 (2007) 011 [hep-ph/0705 . 0116]

[2] A.Rubbia, "Experiments for CP-violation: A giant liquid argon scintillation, Cerenkov and charge imaging experiment?," arXiv:hep-ph/0402110. Invited talk at 2nd International Workshop on Neutrino Oscillations in Venice (NO-VE 2003), Venice, Italy, 3-5 Dec 2003.

[3] S. Amerio et. al. (ICARUS Coll.), Design, construction and tests of the ICARUS T600 detector, Nucl. Instr. Meth. A527 (2004) 329.

[4] L.Oberauer, F.von Feilitzsch and W.Potzel, "A large liquid scintillator detector for low-energy neutrino astronomy,” Nucl. Phys. Proc. Suppl. 138 (2005) 108.

[5] G. Alimonti et. al. (BOREXINO Coll.), The Borexino detector at Laboratori Nazionali del Gran Sasso, Nucl. Instr. Meth. A600 (2009) 568.

[6] A.de Bellefon et al., "MEMPHYS: A large scale water Cerenkov detector at Frejus," arXiv:hepex/0607026.

[7] The Super-Kamiokande Collaboration, The Super-Kamiokande detector, Nucl. Instr. Meth. A501 (2003) 418.

[8] T. Araki et. al. (KamLAND Coll.), Experimental investigation of geologically produced antineutrinos with Kam LAND, Nature 436 (2005) 499. 